\title{
Association of Inflammatory Markers with Mortality in COVID-19 Infection
}

\author{
Abdul Rehman Arshad ${ }^{1}$, Imran Khan², Khurram Shahzad², Murad Arshad², Syed Jarrar Haider ${ }^{2}$ and Muhammad \\ Jawad Aslam² \\ ${ }^{1}$ Department of Nephrology / Medicine, Combined Military Hospital, Peshawar Cantt, Pakistan \\ ${ }^{2}$ Department of Medicine, Combined Military Hospital, Peshawar Cantt, Pakistan
}

\begin{abstract}
Objective: To evaluate the association of serum ferritin, lactate dehydrogenase, and C-reactive protein at admission with inhospital mortality in COVID-19 infection; and to determine best predictive cut-offs.

Study Design: Cross-sectional study.

Place and Duration of Study: Department of Medicine, Combined Military Hospital, Peshawar Cantt; Pakistan from March to June 2020.

Methodology: Admitted patients with SARS-CoV-2 detectable by polymerase chain reaction (PCR) were included. Patients with suggestive radiological findings but negative PCR for SARS-CoV-2, those with incomplete data or those leaving against medical advice were excluded. Serum C-reactive protein, ferritin and LDH levels were tested on admission. SARS-CoV-2 viral load was checked on nasopharyngeal samples. Disease severity was assessed using World Health Organization guidelines.

Results: There were 238 patients, aged $41.18 \pm 16.74$ years. Disease was mild in 157 (65.97\%), moderate in $36(15.13 \%)$, and severe in $45(18.91 \%)$ cases. Twenty-two $(9.24 \%)$ patients died in the hospital. Serum C-reactive protein, ferritin and lactate dehydrogenase levels were elevated in $122(51.26 \%), 83(34.87 \%)$ and $184(77.31 \%)$ patients, respectively; more frequently amongst patients with moderate/severe disease or mortality. Areas under receiver operating characteristic curves and $95 \%$ confidence intervals for serum C-reactive protein, ferritin and LDH were $0.909(0.854-0.964), 0.915(0.835-0.995)$ and 0.863 (0.785-0.942), respectively. C-reactive protein $\geq 45.5 \mathrm{mg} / \mathrm{L}$ had sensitivity $86.36 \%$ and specificity $88.89 \%$; serum ferritin $\geq 723$ $\mathrm{ng} / \mathrm{ml}$ had sensitivity $95.45 \%$ and specificity $86.57 \%$, and lactate dehydrogenase $\geq 428.5 \mathrm{U} / \mathrm{L}$ had sensitivity $90.91 \%$ and specificity $80.56 \%$ for predicting mortality.
\end{abstract}

Conclusion: Levels of the three inflammatory markers at admission can predict mortality in COVID-19 infection.

Key Words: Coronavirus, Inflammation, Mortality, Outcome, Pakistan.

How to cite this article: Arshad AR, Khan I, Shahzad K, Arshad M, Haider SJ, Aslam MJ. Association of Inflammatory Markers with Mortality in COVID-19 Infection. J Coll Physicians Surg Pak 2020; 30(JCPSPCR):CR158-CR163.

\section{INTRODUCTION}

COVID-19 pandemic is a global emergency. Clinical presentation has a wide spectrum ranging from asymptomatic state to florid respiratory failure, requiring ventilatory support. Death is a major concern. As of 6 October 2020, World Health Organization has confirmed 35,274,993 cases and 1,038,534 deaths across 216 countries. ${ }^{1}$ As compared to $2.9 \%$ mortality globally, rate of $2.06 \%$ has been reported by Government of Pakistan. ${ }^{2}$ Inflammation plays a central role in the pathogenesis of COVID-19 infection and its progression.

Correspondence to: Dr. Abdul Rehman Arshad, Department of Nephrology / Medicine, Combined Military Hospital, Peshawar Cantt, Pakistan

E-mail: maj.abdulrehman@gmail.com

Received: August 26, 2020; Revised: October 07, 2020;

Accepted: October 19, 2020

DOI: https://doi.org/10.29271/jcpsp.2020.JCPSPCR.CR158
Activated macrophages release a number of inflammatory cytokine and chemokines, leading to infiltration of neutrophils and monocytes within the lungs. These cause direct injury to alveolar cells and endothelium, causing tissue hypoxia and acute respiratory distress syndrome. ${ }^{3} \mathrm{C}$-reactive protein (CRP) is an important inflammatory marker, often elevated in COVID-19 infection as a protective response. It binds to phosphocholine on the surface of damaged cells, activating the classical complement pathway and thus allowing clearance of virus and damaged cells from the host. ${ }^{4}$

Cytokine storms, characterised by elevated levels of different cytokinesincluding interleukin- 6 and interferon $\gamma$, are the underlying causes of mortality in severe disease. Interleukin- 6 is the major contributor, but not routinely measured at most setups in Pakistan. ${ }^{5} \mathrm{C}$-reactive protein is a reliable substitute and could thus beused todiagnosecytokinerelease syndrome. ${ }^{6}$ Inflammation within the lungs could cause worsening of respiratory failure to an extent requiring invasive ventilation or leading to 
death in as little as three days. ${ }^{7}$ Mortality could be reduced by careful monitoring and follow-up of infected patients, with a view to institute early effective intervention in severe/critical cases. Clinical and laboratory parameters are important in this regard. A number of studies have examined the prognostic role of different inflammatory markers in COVID-19 infection. Many, like procalcitonin and interleukin- 6 are either not widely available or economically unfeasible in developing countries like ours. Serum ferritin, lactate dehydrogenase (LDH) and CRP levels can be determined at almost all laboratories, even in limited resource settings. The results could be of great help in tailoring treatment for individual patients and for following them up.

COVID-19 infection is a relatively new disease. Whereas, the volume of medical literature on this subject is growing rapidly, there is still a dearth of local data examining the relationship between inflammatory markers and clinical outcomes amongst patients with different shades of disease severity. This study was carried out to evaluate the relationship of serum ferritin, LDH and CRP levels at admission with in-hospital mortality amongst patients with COVID-19 infection and to determine cut-off values of the best predictive of mortality.

\section{METHODOLOGY}

This cross-sectional study was carried out at Department of Medicine, Combined Military Hospital, Peshawar Cantt, from March to June 2020. Study protocol was approved by Ethics Review Committee of the Hospital vide reference No. 37. Patients with confirmed COVID-19 infection admitted to the Hospital from March to June 2020 were inducted. Consent from patients was not required because the recorded data could not potentially identify the individuals. Diagnosis was established by a positive real time polymerase chain reaction (PCR) for SARS-CoV-2. Probable cases, defined as patients with findings suggestive of COVID-19 infection on HRCT scan chest but with negative PCRs for SARS-COV-2 on two consecutive occasions were excluded.

Patients with incomplete data on laboratory parameters or those leaving the Hospital against medical advice (LAMA) were also excluded.

Preliminary data from this Hospital revealed mortality rate of $9 \%$ during the initial period of pandemic. A recently conducted study from Pakistan revealed maximum area under curve (AUC) for any of the three inflammatory markers to be $0.875 .{ }^{8}$ Using these parameters, minimum sample size of 192 patients was calculated, including 16 patients with in-hospital mortality and 176 patients getting discharged. This would give $80 \%$ power to detecta difference of 0.2 between curves under null and alternative hypothesis at a significance level of 0.05 .

Nasopharyngeal swabs were taken prior to admission, using standard technique for determination of SARS-CoV-2 by PCR. This was reported qualitatively as being present or absent. As per the Institutional protocol, blood samples were collected within the first half hour of admission for tests including: quanti- tative CRP, serum ferritin and serum LDH. Data on these laboratory parameters as well as duration of hospital stay was collected from the medical paper records. Laboratory information management system software was used to search for missing reports. Primary outcome was death in hospital. Disease severity was assessed using World Health Organization guidelines (Table I). ${ }^{9}$ Considering only a very few patients with critical disease at presentation, they were merged into the severe diseasegroup.

Table I: Clinical severity of COVID-19 infection (adapted from World Health Organization interim guidance on clinical management of COVID-19). ${ }^{9}$

\begin{tabular}{|l|l|}
\hline Category & Definition \\
\hline Mild & $\begin{array}{l}\text { Symptomatic patients fulfilling case definition for } \\
\text { COVID-19 without evidence of pneumonia or } \\
\text { hypoxia. }\end{array}$ \\
\hline Moderate & $\begin{array}{l}\text { Clinical signs of pneumonia (fever, cough, dyspnoea) } \\
\text { but no signs of severe pneumonia, including SpO2 } \\
\geq 90 \% \text { on room air. }\end{array}$ \\
\hline Severe & $\begin{array}{l}\text { Clinical signs of pneumonia (fever, cough, dyspnoea) } \\
\text { plus one of the following: respiratory rate }>30 \\
\text { breaths/min; severe respiratory distress; or SpO2 } \\
<90 \% \text { on room air. }\end{array}$ \\
\hline Critical & \begin{tabular}{l} 
Acute respiratory distress syndrome. \\
\hline
\end{tabular} \\
\hline
\end{tabular}

Table II: Clinical characteristics.

\begin{tabular}{|c|c|c|}
\hline \multicolumn{2}{|l|}{ Variable } & Value \\
\hline \multicolumn{2}{|l|}{ Age (years) } & $41.18 \pm 16.74$ \\
\hline \multirow{2}{*}{ Gender } & Male & $208(87.39 \%)$ \\
\hline & Female & 30 (12.61\%) \\
\hline \multirow{3}{*}{$\begin{array}{l}\text { Disease severity at } \\
\text { admission }\end{array}$} & Mild & $157(65.97 \%)$ \\
\hline & Moderate & $36(15.13 \%)$ \\
\hline & Severe/critical & $45(18.91 \%)$ \\
\hline \multicolumn{2}{|c|}{ Duration of hospital stay (days) } & $11.06 \pm 5.70$ \\
\hline \multirow{4}{*}{ Mortality* } & Total & $22(9.24 \%)$ \\
\hline & Mild disease & $0(0.00 \%)$ \\
\hline & Moderate disease & $2(9.09 \%)$ \\
\hline & Severe/critical disease & $20(90.91 \%)$ \\
\hline
\end{tabular}

Data was analysed using IBM SPSS Statistics for Windows, Version 20.0 (IBM Corp, Armonk, NY). Qualitative data were expressed as numbers and percentages. Quantitative data was described as mean \pm standard deviation. Median and interquartile range were used for continuous variables with non-parametric distribution. Normality of data was checked using Shapiro-Wilk test. Kruskal-Wallis test was done to compare median and interquartile ranges for different inflammatory markers amongst patients with mild, moderate, and severe disease.

Amongst the two groups based on outcomes, levels of inflammatory markers were compared using Independent-samples Mann-Whitney U-test. Proportions of patients with elevated levels of inflammatory markers amongst different groups were compared using Chi-square/ Fisher's exact test. Receiver operating characteristic (ROC) curve analysis was carried out to determine the sensitivity and specificity of the three inflammatory markers for predicting mortality and to determine the most appropriate cut-off values. AUC for different inflammatory markers were automatically reported by IBM SPSS Statistics, 
while generating the curves. This parameter is a measure of how good the statistical model is at differentiating between different classes. For example, a higher AUC for a given inflammatory marker in this study would mean better differentiation between people dying in hospital or getting discharged. KaplanMeier method was used to determine the probability of getting discharged from hospital as predicted by different inflammatory markers at these thresholds. For all comparisons, $p<0.05$ was considered significant. Dataset for this study is available online in Harvard Dataverse. ${ }^{10}$

\section{RESULTS}

During this study period, 431 patients with COVID-19 infection were admitted to this hospital. Thirty-six died during hospital stay, giving a mortality rate of $8.35 \%$. Complete results for inflammatory markers could not be traced for 117 patients, 73 had probable COVID-19 infection, while three left against medical advice. They were thus excluded from data analysis. Clinical characteristics of the remaining 238 patients included in this study are shown in Tablell.

None of the patients with mild disease died. Absolute values of inflammatory markers for the study population are also presented (Table III). Serum CRP, ferritin and LDH levels were elevated in 122 (51.26\%), 83 (34.87\%) and 184 (77.31\%) patients, respectively.

These parameters were above upper limits of normal in a greater proportion of patients with moderate to severe disease or those dying in hospital (Figure 1).

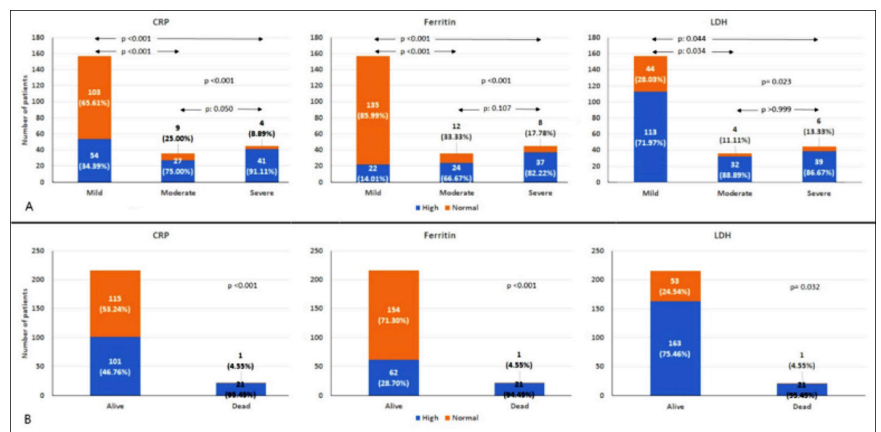

Figure 1: Comparison of proportions of patients with elevated inflammatory markers, based on clinical disease severity (Panel A) and outcomes (PanelB).

AUC and 95\% confidence intervals for serum CRP, ferritin and LDH were 0.909 (0.854-0.964), 0.915 (0.835-0.995) and 0.863 (0.785- 0.942), respectively (Figure 2). Evaluation of different coordinates for these curves revealed that CRP $\geq 45.5 \mathrm{mg} / \mathrm{l} \mathrm{had}$ sensitivity of $86.36 \%$ and specificity of $88.89 \%$ for predicting mortality. Similarly, serum ferritin $\geq 723 \mathrm{ng} / \mathrm{ml}$ at admission had a sensitivity of $95.45 \%$ and specificity of $86.57 \%$ for predicting mortality; whereas, $\mathrm{LDH} \geq 428.5 \mathrm{U} / \mathrm{l}$ had sensitivity of $90.91 \%$ and specificity of $80.56 \%$ for predicting mortality. Kaplan-Meier survival analysis as predicted by different inflammatory markers at these thresholds is shown in Figure 3.

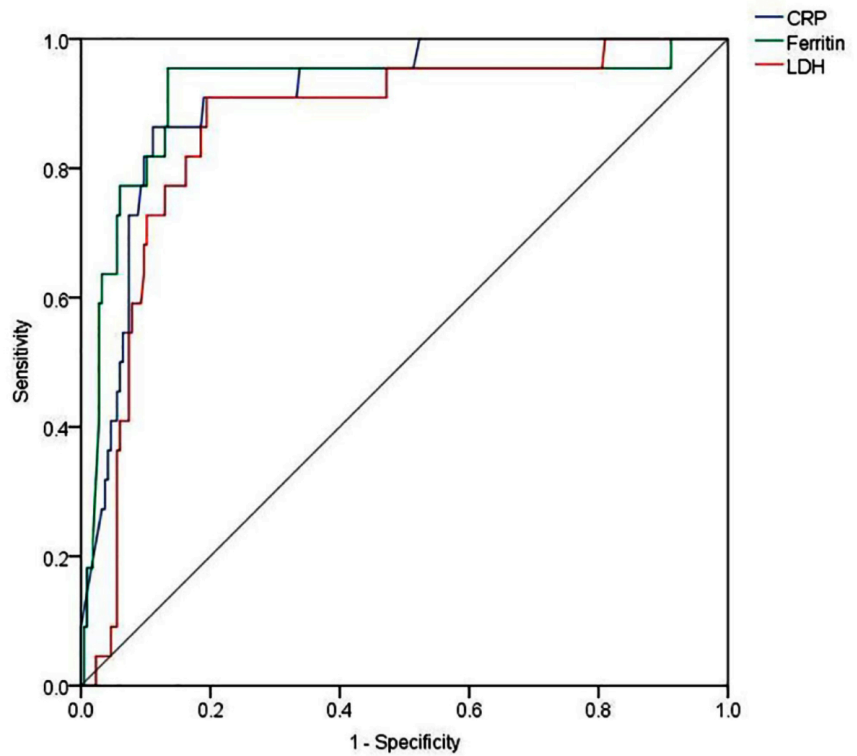

Figure 2: ROC curves for different inflammatory markers.
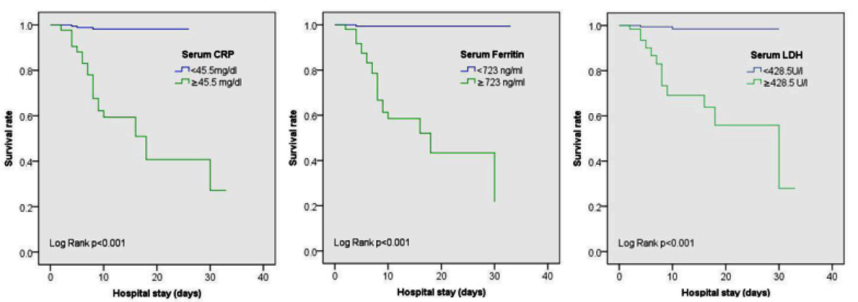

Figure 3: Kaplan-Meier survival curves according to levels of inflammatory makers atadmission.

\section{DISCUSSION}

These results highlight the higher levels of inflammatory markers amongst patients with moderate to severe disease, as compared to milder forms. While this is in keeping with the currently available evidence and the concept of cytokine storm as a pathophysiological model for severe COVID-19 infection, there was no difference amongst patients with moderate and severe disease with regard to inflammatory markers. The findings are in contrast to other available literature, where patients with severe disease have higher inflammatory markers as compared to those with moderate disease. ${ }^{11}$ These results might simply be because of moderate and severe categories being towards the upper end of severity spectrum. Indeed, patients with moderate to severe disease are recommended treatment in hospital; whereas, those with mild disease could be observed at home. ${ }^{12}$ A study done in USA failed to show any difference in CRP levels in moderate and severe disease. ${ }^{13}$

Of all the inflammatory markers, serum ferritin levels were elevated in least proportions of patients with moderate/severe disease. Yet, serum ferritin outperformed other inflammatory markers in predicting mortality, with greatest AUC and odds ratio. High serum ferritin levels were also independently associated with poor outcomes in the patients of this study. 
Table III: Comparison of inflammatory markers amongst different groups.

\begin{tabular}{|c|c|c|c|c|c|c|c|c|}
\hline & \multirow{2}{*}{$\begin{array}{l}\text { All patients } \\
\qquad(n=238)\end{array}$} & \multicolumn{3}{|c|}{ Outcomes } & \multicolumn{4}{|c|}{ Clinical disease severity } \\
\hline & & $\begin{array}{l}\text { Discharged } \\
(n=216)\end{array}$ & $\begin{array}{c}\text { Death } \\
(n=22)\end{array}$ & $\boldsymbol{P}$ & $\begin{array}{c}\text { Mild } \\
(n=157)\end{array}$ & $\begin{array}{c}\text { Moderate } \\
(n=36)\end{array}$ & $\begin{array}{l}\text { Severe } \\
(n=45)\end{array}$ & $\boldsymbol{p}$ \\
\hline CRP (mg/l) & $\begin{array}{c}6.15 \\
(2.18-29.13) \\
\end{array}$ & $\begin{array}{c}4.50 \\
(2.03-21.00)\end{array}$ & $\begin{array}{c}120.50 \\
(74.75-180.00)\end{array}$ & $<0.001$ & $\begin{array}{c}3.00 \\
(1.55-7.50)\end{array}$ & $\begin{array}{c}24.50 \\
(5.10-53.25)\end{array}$ & $\begin{array}{c}91.00 \\
(27.30-146.50)\end{array}$ & $<0.001$ \\
\hline $\begin{array}{l}\text { Ferritin } \\
\text { (ng/ml) }\end{array}$ & $\begin{array}{c}232.00 \\
(118.00-603.75)\end{array}$ & $\begin{array}{c}215.00 \\
(109.00-439.25)\end{array}$ & $\begin{array}{c}1759.50 \\
(1198.75-2100.50)\end{array}$ & $<0.001$ & $\begin{array}{c}171.00 \\
(98.00-278.50)\end{array}$ & $\begin{array}{c}471.50 \\
(261.75-837.50)\end{array}$ & $\begin{array}{c}1276 \\
(611.50-1915.00) \\
\end{array}$ & $<0.001$ \\
\hline LDH (U/I) & $\begin{array}{c}300 \\
(227.5-435.5)\end{array}$ & $\begin{array}{c}288.00 \\
(225.00-402.25)\end{array}$ & $\begin{array}{c}640.00 \\
(533.50-804.50)\end{array}$ & $<0.001$ & $\begin{array}{c}265 \\
(221-352)\end{array}$ & $\begin{array}{c}409.00 \\
(263.75-600.50)\end{array}$ & $\begin{array}{c}493.00 \\
(349.50-712.50)\end{array}$ & $<0.001$ \\
\hline
\end{tabular}

It is believed that the high ferritin levels in COVID-19 infection are driven by increased production driven by proinflammatory cytokines (interleukin- 6 and tumor necrosis factor- $\alpha$ ) or release from damaged cells. ${ }^{14}$ In a study done by Feld et al. serum ferritin at admission was a poor predictor of all-cause mortality, with an AUC of $0.677 .{ }^{15}$ The optimal cut-off level was $799 \mathrm{ng} / \mathrm{ml}$ for this purpose. However, African-American patients with elevated ferritin levels were almost two times more likely to die in New York, consistent with the findings of this study. ${ }^{16}$ Serum ferritin levels have also been used to predict disease severity and complications. Herold et al. documented an AUC of 0.750 to predict need for mechanical ventilation using a cut-off of $1285 \mathrm{ng} / \mathrm{mll}^{17}$

A minimum seven-fold increase in CRP levels was best predictive of mortality in these patients. Different cut-offs for CRP have been described for complications/death in various studies. A meta-analysis by Huang et al. described nearly three times increased risk of mortality amongst patients with $\mathrm{CRP} \geq 10 \mathrm{mg} / \mathrm{L} .{ }^{18}$ Liu et al. showed that CRP $>41.8 \mathrm{mg} / \mathrm{L}$ was associated with severe complications. ${ }^{19}$ In a study by Luo et al. CRP $>41.4 \mathrm{mg} / \mathrm{L}$ was $90.5 \%$ sensitive and $77.6 \%$ specific for mortality amongst hospitalised patients. ${ }^{20}$ Raised LDH levels, a marker of cell damage, have previously been reported in other viral infections like MERS-CoV and $H 5 N 1{ }^{21}$ ROC curve analysis revealed an almost two times rise foretelling death during hospitalisation in this cohort. Patients with $\mathrm{LDH}$ levels $\geq 1200 \mathrm{U} / \mathrm{L}$ in an American study were eight times more likely to die. ${ }^{22}$ Elevated CRP and LDH could thus help alert physicians to the possibility of guarded prognosis.

Asghar et al. analysed data on 364 indoor patients with COVID-19 infection from Pakistan. ${ }^{8}$ Besides many other laboratory parameters, they evaluated the three inflammatory markers used in this study as well. All of them had lower AUC as compared to the present results. Interestingly, serum ferritin was the weakest predictor in their data set. While this may just be a co-incidence, we would suggest using serum ferritin as a first priority based on our experience.

Males outnumbered females in this study, with a similar gender distribution across different classes of disease severity. Data from Global Health 50/50 Research Initiative shows $74 \%$ of confirmed COVID-19 cases from Pakistan to be males. ${ }^{23}$ This is in contrast to European countries providing gender-disaggregated data, where there is no major gender-based difference in the absolute number of confirmed cases. ${ }^{24}$ The figures of this study are most probably a reflection of the conservative society in this geographical area. Males enjoy greater freedom of movement outside homes and are thus more prone to acquire COVID-19 infection.

An important strength of this dataset is that blood sampling for inflammatory markers was done within half an hour of admission, as per the policy of the study centre. Patients with missing parameters from the day of admission were excluded, thus avoiding bias. While the authors only studied the impact of inflammatory markers measured at the time of admission, it would be interesting to see how changes during hospitalisation affect the outcomes. The authors could not check levels of other inflammatory markers such as procalcitonin and interleukin- 6 in majority of patients due to financial constraints. A standardised format for documenting medical notes was not followed during this period of excessive workload. The authors were thus not able to capture information about various factors that could have adversely affected inflammatory markers, especially CRP. Latter is often lower in patients with cirrhosis or those on medications such as statins. ${ }^{25}$ The results of this study should be interpreted in context of its retrospective nature and the fact that some patients had to be excluded because of missing data. It is very much possible that these patients did not have testing done on the day of admission because of perceived mild severity of illness. Mortality rate in this study group was also a little higher than the entire cohort treated at the Hospital. Still, the results provide a very useful insight into the ability of the three inflammatory markers to predict in-hospital death. The authors would suggest multi-centre studies incorporating larger number of patients to refine the results further.

\section{CONCLUSION}

Serum CRP, ferritin, and LDH levels at the time of admission can predict mortality in COVID-19 infection. Serum ferritin is the best amongst them for this purpose. Relevant information is readily available on the day of admission, when many patients have not actually deteriorated. This should give clinicians a great opportunity to closely monitor patients with 
high levels of inflammatory markers and institute treatment measures to prevent poor outcomes.

\section{ETHICAL APPROVAL:}

The study protocol was approved by Ethics Review Board of Combined Military Hospital Peshawar prior to initiation of data collection (Approval No. 37, dated 4 July, 2020).

\section{PATIENTS' CONSENT:}

Consents were not taken from all patients as no information that could potentially identify the patients was recorded.

\section{CONFLICT OF INTEREST:}

None to be declared by any of the authors.

\section{AUTHORS' CONTRIBUTION:}

ARA: Designed the study, acquired data, critically revised the manuscript, approved the final version, agreed to be accountable for all aspects.

IK: Conceived the study, analysed data, drafted the manuscript, approved the final version, agreed to be accountable for all aspects.

KS: Interpreted data, drafted the manuscript, approved the final version, agreed to be accountable for all aspects.

MA, SJH, MJA: Analysed data, drafted the manuscript, approved the final version, agreed to be accountable for all aspects.

\section{REFERENCES}

1. WHO Coronavirus Disease (COVID-19) Dashboard. World Health Organization. http://covid19.who.int/. (accessed on $6 / 10 / 2020$ )

2. COVID-19 Health Advisory Platform by Ministry of National Health Services Regulations \& Coordination. Government of Pakistan. http://covid.gov.pk/stats/ pakistan (accessed on $6 / 10 / 2020$ )

3. Lingeswaran M, Goyal T, Ghosh R, Suri S, Mitra P, Misra S, et al. Inflammation, immunity and immunogenetics in COVID-19: A narrative review. Indian J Clin Biochem 2020; 35(3):260-73. doi: 10.1007/s12291-020-00897-3.

4. Ali N. Elevated level of C-reactive protein may be an early marker to predict risk for severity of COVID-19. J Med Virol 2020; doi10.1002/jmv.26097.

5. Chen X, Zhao B, Qu Y, Chen Y, Xiong J, Feng Y, et al. Detectable serum SARS-CoV-2 viral load (RNAaemia) is closely correlated with drastically elevated interleukin 6 (IL-6) level in critically ill COVID-19 patients. Clin Infect Dis 2020; 10.1093/cid/ciaa449.

6. Lee DW, Gardner R, Porter DL, Louis CU, Ahmed N, Jensen $M$, et al. Current concepts in the diagnosis and management of cytokine release syndrome. Blood 2014; 124(2):188-95. doi: 10.1182/blood-2014-05-552729.

7. Vultaggio A, Vivarelli E, Virgili G, Lucenteforte E, Bartoloni A, Nozzoli C, et al. Prompt predicting of early clinical deterioration of moderate-to-severe COVID-19 patients: Usefulness of a combined score using IL- 6 in a preliminary study. J Allergy Clin Immunol Pract 2020; 8(8):2575-81. doi: 10.1016/j.jaip.2020.06.013.

8. Asghar M, Kazmi SJH, Khan NA, Akram M, Hassan M, Rasheed $U$, et al. Poor prognostic biochemical markers predicting fatalities caused by COVID-19: A retrospective observational study from a developing country. Cureus 2020; 12(8):e9575. doi: 10.7759/cureus.9575.

9. Clinical management of COVID- 19: interim guidance, 27 May. World Health Organization. http://apps.who.int/iris/ handle/10665/332196. (accessed on 12/7/2020).

10. Arshad AR, "Inflammatory markers in COVID-19 pneumonia", Harvard Dataverse, V1, 2020, http://doi.org/ 10.7910/DVN/ JGUOUM.

11. Zeng Z, Yu H, Chen H, Qi W, Chen L, Chen G, et al. Longitudinal changes of inflammatory parameters and their correlation with disease severity and outcomes in patients with COVID-19 from Wuhan, China. Crit Care 2020; 24(1):525. doi: 10.1186/s13054-020-03255-0.

12. Gandhi RT, Lynch JB, Del Rio C. Mild or moderate Covid-19. N Engl J Med 2020; Doi. 10.1056/NEJMcp 2009249.

13. Miatech JL, Yaslik CP, Tarleton HE, West D, Kellum W, McKnight $M$, et al. Retrospective analysis of inflammatory markers and patient characteristics in hospitalised Covid-19 patients: an early experience in Louisiana. Cureus 2020; 12(9):e10257. doi: 10.7759/ cureus.10257.

14. Lin Z, Long F, Yang $Y$, Chen $X, X u$ L, Yang M. Serum ferritin as an independent risk factor for severity in COVID-19 patients. J Infect 2020; 81(4):647-79. doi: 10.1016/j.jinf.2020.06.053

15. Feld J, Tremblay D, Thibaud S, Kessler A, Naymagon L. Ferritin levels in patients with COVID-19: A poor predictor of mortality and hemophagocytic lymphohistiocytosis. Int J Lab Hematol 2020; 10.1111/ijlh.13309.

16. Gayam V, Chobufo MD, Merghani MA, Lamichanne S, Garlapati PR, Adler MK. Clinical characteristics and predictors of mortality in African-Americans with COVID-19 from an inner-city community teaching hospital in New York. J Med Virol 2020; doi. 10.1002/jmv.26306.

17. Herold T, Jurinovic V, Arnreich C, Lipworth BJ, Hellmuth JC, von Bergwelt-Baildon $M$, et al. Elevated levels of IL- 6 and CRP predict the need for mechanical ventilation in COVID-19. J Allergy Clin Immunol 2020; 146(1):128-36. e124. doi: 10.1016/j.jaci.2020.05.008.

18. Huang I, Pranata R, Lim MA, Oehadian A, Alisjahbana B. Creactive protein, procalcitonin, $D$-dimer, and ferritin in severe coronavirus disease-2019: A meta-analysis. Ther Adv Respir Dis 2020; 14:1753466620937175.

19. Liu F, Li L, Xu M, Wu J, Luo D, Zhu Y, et al. Prognostic value of interleukin-6, C-reactive protein, and procalcitonin in patients with COVID-19. J Clin Virol 2020; 127:104370. doi: 10.1016/j.jcv.2020.104370.

20. Luo X, Zhou W, Yan X, Guo T, Wang B, Xia H, et al. Prognostic value of $C$-reactive protein in patients with COVID-19. Clin Infect Dis 2020; ciaa641. doi: 10.1093/ cid/ciaa641.

21. Shi J, Li Y, Zhou X, Zhang Q, Ye X, Wu Z, et al. Lactate dehydrogenase and susceptibility to deterioration of mild 
COVID-19 patients: A multicenter nested case-control study. BMC Med 2020; 18(1):168. doi: 10.1186/s12916020-01633-7.

22. Ayanian S, Reyes J, Lynn L, Teufel K. The association between biomarkers and clinical outcomes in novel coronavirus pneumonia in a US cohort. Biomark Med 2020; 14(12): 1091-7. Doi. 10.2217/bmm-2020-0309.

23. The COVID-19 sex-disaggregated data tracker. Global Health 5050. http://globalhealth5050.org/the-sex-gender- and-covid-19-project/the-data-tracker/? explore=country \&country=Pakistan\#search. (accessed on 6/10/2020)

24. Gebhard C, Regitz-Zagrosek V, Neuhauser HK, Morgan R, Klein SL. Impact of sex and gender on COVID-19 outcomes in Europe. Biol Sex Differ 2020; 11(1):29. doi: 10.1186/ s13293-020-00304-9.

25. Asher J, Houston M. Statins and C-reactive protein levels. J Clin Hypertens (Greenwich) 2007; 9(8):622-8. doi.org/ 10.1111/j.1524-6175.2007.06639.x. 